ORIGINAL ARTICLE

\title{
Clinico-demographic profile of patients treated for Tuberculous Lymphadenitis at the Central Chest Clinic, Colombo, Sri Lanka
}

\author{
Nadeesha Amarasinghe ${ }^{1}$, Amitha Fernando ${ }^{1}$, Aflah Sadikeen ${ }^{1}$, Thilakshi Perera ${ }^{1}$, Ghazzaly \\ Nizamdeen $^{1}$, Deepal Jayamanne ${ }^{2}$, Sawani Nanayakkara ${ }^{3}$ \\ ${ }^{1}$ Central Chest Clinic, Colombo, Sri Lanka \\ ${ }^{2}$ Faculty of Medicine, University of Kelaniya, Sri Lanka \\ ${ }^{3}$ Medical Research Institute, Colombo, Sri Lanka
}

\section{Article Information}

Total number of

Words 2350

Tables 02

Authors have no conflicts of interest to declare

Keywords: Tuberculous lymphadenitis, Extra pulmonary tuberculosis, Paradoxical reactions

Date of submission:04.04.2018

Date of acceptance: 20.11 .2018

Author responsible for correspondence: Nadeesha Amarasinghe Central Chest Clinic, Colombo 384/5A, Kapuwadeniya Road, Thalangama North

E mail: amaranadee@gmail.com

http://orcid.org/0000-0001-6620-1743

DOI: http://doi.org/10.4038/cjms. v55i2.4955

\begin{abstract}
Background:

Tuberculous lymphadenitis (TBLN) is the commonest form of extrapulmonary tuberculosis in Sri Lanka. The management of TBLN faces many challenges due to difficulty in achieving microbiological confirmation as well as slow or paradoxical clinical response during the treatment.
\end{abstract}

\section{Objective:}

To describe the demographic features and the characteristics associated with clinical presentation, diagnostic modalities and treatment outcome of patients with TBLN.

\section{Method:}

An audit was carried out revising clinical records of patients treated for peripheral lymph node enlargement due to tuberculosis at the Central Chest Clinic, Colombo.

\section{Results:}

A total of 126 clinical records were analysed. The male:female ratio was $1: 1.6$ with a mean age of 36 years $(\mathrm{SD}=17.6)$. The most affected site were the cervical lymph nodes (94\%). Mantoux test positivity $(>10 \mathrm{~mm})$ was detected in $87.5 \%$. Evidence of granulomatous inflammation in primary investigations was seen in $122(97 \%)$ of patients. Out of all only $12(9.5 \%)$ were microbiologically confirmed including 5 culture positives. The rest of the patients $(90.5 \%)$ were diagnosed based on supportive primary and ancillary diagnostic tests. Paradoxical reactions were identified in $4 \%$ of patients. Adverse drug reactions including skin reactions and drug induced hepatitis were noted in 17\%. Eighty-two patients achieved complete clinical resolution at the end of 6 months while others were found to have residual lymph nodes necessitating further evaluation.

\section{Conclusions:}

The incidence of TBLN was high among females and in the third decade of life, predominantly affecting cervical lymph nodes. The majority were treated based on primary and ancillary diagnostic test results. Prospective studies are recommended for more descriptive assessment.

\section{Background}

Out of all forms of Tuberculosis, Tuberculous Lymphadenitis (TBLN) remains the commonest form of extra-pulmonary tuberculosis reported worldwide[1-4]. It is the same in Sri Lanka as well with 723 patients with TBLN noted in 2015[4].

The definitive diagnosis of TBLN is achieved by culture or polymerase chain reaction (PCR) of $\mathrm{M}$. tuberculosis in affected nodes. Positive culture remains the gold standard[5]. Excisional biopsy is 
an invasive approach but carries a higher sensitivity. The histology of these nodes characteristically shows granuloma formation by epithelioid histiocytes and Langerhans type Giant cells with or without caseation necrosis[6,7]. In spite of their usefulness, availability of these investigations is limited due to the facilities available, time taken and cost[8]. Fine needle aspiration (FNAC) on the other hand is a minimally invasive, time-saving investigation in most resource-limited settings[6]. Hematoxylin \& Eosin staining of aspirates would demonstrate granulomata as aggregates of histiocytes and associated multinucleated giant cells while a dirty necrotic background would suggest caseation $[6,7,9]$.

Presence of fever and constitutional symptoms are not documented as a common presenting complaint in TBLN[10]. However, when lymphadenopathy reveals granulomatous inflammation with positive ancillary test results such as Mantoux test and raised Erythrocyte sedimentation rate (ESR), in most tuberculosis (TB) prevalent settings the practice is to start on anti-tuberculous treatment (ATT) without spending time on a extensive workup to exclude other causes for granulomatous inflammation such as fungal infections, infections by non-tuberculous mycobacterium, sarcoidosis, Crohn and Wegener disease etc.[6].The standard treatment duration is 6 months of anti TB drugs but continuation up to 9 months is considered in some settings[10-15]. Management of TBLN poses many challenges due to slow or paradoxical clinical responses such as appearance of freshly involved nodes, enlargement of the existing nodes, development of fluctuation, appearance of sinus tracts and residual lymphadenopathy after completion of treatment [16].

Demographic and clinical characteristics of patients can vary based on the geographical setting. Knowledge on previously treated patients will lead to confident decision making specially in doubtful situations. This audit was conducted to understand the clinico-demographic profiles of patients treated as for TBLN in Sri Lanka.

\section{Methods}

A retrospective audit was carried out reviewing medical records of patients treated for TBLN during 2015-16 two year period at the Central Chest Clinic, Sri Lanka. Those who had not completed the full course of treatment were excluded.

The frequency of TB lymphadenitis was analysed according to the gender, age and the ethnicity. Clinical presentation including the site of lymph node involvement, associated constitutional symptoms, presence of co-morbidities and the results of the microbiological investigations, primary and ancillary investigations and the treatment outcome were recorded. Data were analysed using statistical package for the social sciences (SPSS) version 22.0. Data were summarized using descriptive statistics. Ethical clearance was obtained from the Ethics Review committe of the Medical Research Institute, Sri Lanka.

\section{Results}

A total of 126 complete clinical records were analysed. Male: female ratio was 1:1.6. The highest frequency of TBLN for age was seen in the third decade of life (mean age $=36$ years, $\mathrm{SD}=17.6$ ). The distribution according to Sinhala, Tamil and Moor ethnic groups were $71 \%, 15 \%$ and $14 \%$ respectively and did not show a significant difference between the percentages $(\mathrm{P}>.05)$ when compared with the percentages of ethnic distribution in Colombo District[17].

The presenting complaint and the frequency of involvement of different lymph node groups are illustrated in Table 1. Diabetes was the commonest (6\%) co-morbidity in this group. One patient had HIV infection. The majority $(\mathrm{n}=108,86 \%)$ did not have co-morbidities. The Mantoux test was positive $(>10 \mathrm{~mm})$ in $96(87.2 \%)$ individuals and the result was not recorded in 16 files. The ESR was documented to be high in $116(92 \%)$. Chest radiographs of all 126 patients were normal. Compatible ultra sound reports were available in 22 patients. All patients had undergone a FNAC, lymph node biopsy or both as primary diagnostic investigations and the findings are illustrated in Table 2. Evidence of granulomatous inflammation was identified in 122 (97\%) of patients. Out of them $74(60.6 \%)$ were caseating granuloma type and 48 $(39.3 \%)$ were non caseating granuloma type. TB culture reports were available only in 14 patients of which $5(35.7 \%)$ only were positive. Reports of smears for Acid Fast Bacilli (AFB) were available in 9 patients and of them 7 (78\%) were positive. Out of 10 samples sent for polymerase chain reaction (PCR), only 4 were positive.

Out of 126 patients studied only $12(9.5 \%)$ were microbiologically confirmed and the diagnosis of TBLN in the rest (90.5\%) was based on supportive primary and ancillary diagnostic tests. The presence of granulomatous inflammation with a positive Mantoux test was the minimal diagnostic criteria used to start ATT. There was no significant difference between ESR positivity with granulomatous inflammation without caseation and granulomatous inflammation with caseation $(\mathrm{P}>0.05)$.

There were no details on parallel investigations 
to exclude other causes for granulomatous inflammation found in the records. Paradoxical reactions including further enlargement of persisting nodes and development of new nodes during the treatment were identified in $5(4 \%)$ patients. Adverse drug reactions were identified in 21 (17\%) patients after commencement of ATT. These were 17 with skin reactions and 4 with laboratory confirmed drug induced hepatitis.

Complete clinical resolution at the end of 6 months was achieved in $65 \%$ including those with microbiological confirmation of diagnosis. Thirty patients out of 44 patients with incomplete resolution were subjected to an extended treatment (total 9 months). Those not offered extended treatment due to small residual node size when reassessed after 3 months and had no progression of nodular size.

Table 1. Clinical characteristics at presentation

\begin{tabular}{ll}
\hline Characteristic & Frequency \\
\hline Presentation & $113(89.7 \%)$ \\
Painless lump & $9(7.1 \%)$ \\
Lump + LOA and LOW & $2(1.6 \%)$ \\
Lump + Fever & $2(1.6 \%)$ \\
Lump+ Fever + LOW and LOA & \\
\hline Site of lymph node enlargement & $115(91.3 \%)$ \\
Cervical & $4(3.2 \%)$ \\
Cervical + Axillary & $4(3.2 \%)$ \\
Axillary & $2(1.6 \%)$ \\
Inguinal & $1(0.8 \%)$ \\
Parotid &
\end{tabular}

LOA-Loss of appetite LOW-Loss of weight

Table 02. Results of FNAC and LN biopsy

\begin{tabular}{lll}
\hline Result & $\begin{array}{l}\text { FNAC } \\
\text { n=59 } \\
\text { Frequency }\end{array}$ & $\begin{array}{l}\text { LN biopsy } \\
\text { n=80 } \\
\text { Frequency }\end{array}$ \\
\hline GI with caseation & $22(37.3 \%)$ & $53(66.2 \%)$ \\
GI without caseation & $27(45.8 \%)$ & $25(31.2 \%)$ \\
Abscess & $5(8.5 \%)$ & $1(1.2 \%)$ \\
Reactive changes & $5(8.5 \%)$ & $1(1.2 \%)$ \\
\hline
\end{tabular}

FNAC-Fine needle aspiration cytology

LN - Lymph node

GI- Granulomatous inflammation

\section{Discussion}

The audit describes the clinico-demographic characteristics of patients treated for TBLN at the Central Chest Clinic. The revealed high incidence of TBLN among females is similar to studies conducted in TB endemic as well as Non-TB endemic countries[5,10,16,18]. It is reported that the prevalence is higher in the younger population, although the decade of highest incidence presented varies among countries[10,19]. Therefore, the characteristics described, related to age and gender in this study is compatible with other countries. Presentation with a painless lump predominantly of cervical lymph nodes in this study is also comparable with the literature $[5,10,14]$. Outstanding difference in comparison to studies from elsewhere, is that most of our patients were immunocompetent in contrast to high incidence of patients with HIV reported from some other countries[16-20]. Presence of constitutional symptoms and fever is not documented as a common presenting complaint with TBLN in our study and is in agreement with other publications[10]. Lymph nodes were of caseating granuloma type in $74(58.7 \%)$ and noncaseating granuloma type in $48(38.1 \%)$. This finding is compatible with the percentages in previous studies[21,5].

Availability of culture reports which leads to a definitive diagnosis was highly insufficient. Majority of the patients were referred to the chest clinic after completion of initial investigations from referring health care units. Further search revealed that specific TB culture request forms and culture bottles are not freely available at primary investigation units and sending specimens for TB culture during lymph node biopsy and FNAC procedures has not been done due to practical limitations. This finding highlights the need to optimize the facilities available for microbiological investigations for $\mathrm{TB}$ in the units where initial investigations are carried out. However, patients being treated based on FNAC or histological findings supported by ancillary tests remains the practice in most TB endemic countries[10]. The incidence of paradoxical reactions reported was low compared to previous publications[10]. There was no documented diagnostic work up to re-evaluate patients who had residual lymphadenopathy at the end of standard treatment regimen and this is an area to be addressed in future research.

\section{Conclusion and recommendations}

In this cohort of patients with TBLN, the frequency was higher among females and those in the third decade of life. They had predominantly affected the cervical lymph nodes. The majority were treated based on primary and ancillary diagnostic tests. Availability of confirmative evidence was highly insufficient.

Primary investigation units should be promoted to send samples for TB culture when performing suspected lymph node biopsy and FNAC in patients with TBLN. Future audits and prospective studies on 
diagnostic workup and treatment outcomes will be useful to enhance patient care. A common checklist will minimize the shortcomings in documentation.

\section{Acknowledgement}

Authors acknowledge contribution by Dr Lakmali Amarasiri, Dr A U Jayasinghe and all other staff members of the Central Chest Clinic.

\section{References:}

1. The World Health Organization Regional Office of South East Asia. Tuberculosis Control in South-East Asia Region. WHO Regional office for South- East Asia. Annual report 2015. http://www.who.int/ iris/handle/10665/ 206062 (accessed 05 Jan 2018)

2. Mohapatra PR, Janmeja AK. Tuberculous lymphadenitis. J Assoc Physicians India 2009;57:585-90.

3. Agab Eldour AA, Mohmed Salih EN et al. Incidence of Tuberculosis and Amyloidosis among Sudanese patients presented with enlarged nodes. J Trop Med 2014; 2014:832029. doi:10.1155/2014/832029

4. National control programme for Tuberculosis and chest diseases. Annual Report - 2015. Ministry of Health, Sri Lanka 2017.

5. Ahmed HGE, Nassar AS, Ginawi I. Screening for Tuberculosis and its histological pattern in patients with enlarged lymph node. Pathology Res Int 2011;2011:417635. doi:10.4061/2011/417635

6. Majeed MM, Bukhari MH. Evaluation for granulomatous inflammation on fine needle aspiration cytology using special Stains. Pathology Res Int 2011;2011:851524. doi:10.4061/851524.

7. Bezabih M, Mariam DW, Selassie SG. Fine needle aspiration cytology of suspected tuberculous lymphadenitis. Cytopathology 2002;13:284-290. doi:10.4061/j.1365-2303. 2002. 00418x.

8. Koo V, Lioe T, Spence R. Fine needle aspiration cytology (FNAC) in the diagnosis of granulomatous lymphadenitis. Ulster Med J 2006;75:59-64.

9. Fontanilla JM, Barnes A, von Reyn CF. Current diagnosis and management of peripheral Tuberculous Lymphadenitis. Clinic Infect Dis 2011;53:555-62. doi/10.1093/cid/cir454.

10. World Health Organization, Stop TB Initiative (World Health Organization), editors. Treatment of tuberculosis: guidelines. 4th ed. Geneva: World Health Organization 2010:147. https://www.who. int/tb/publications/9789241547833/en/ (accessed 06 Feb 2018).
11. Nahid P, Dorman SE, Alipanah N et al. Official American Thoracic Society/ Centres for Disease Control and Prevention/Infectious Diseases Society of America Clinical Practice Guidelines: Treatment of Drug - Susceptible Tuberculosis. Clinic Infect Dis 2016;63:853-867. doi:10.1093/cid/ciw376

12. National Institute for Health and Care Excellence. Tuberculosis 2016. https://www. nice.org.uk/guidance/ng33/resources/tuberculosi 1837390683589 (accessed 01 March 2018).

13. Cook VJ, Manfreda J, Hershfield ES. Tuberculous Lymphadenitis in Manitoba: Incidence, clinical characteristics and treatment. Can Resp J 2004;11:279-86.

14. National Control Programme for Tuberculosis Control and Chest Diseases. Extrapulmonary Tuberculosis- A guideline for the chest clinics and other health settings. Ministry of Health, Sri Lanka 2013.

15. Gupta PR. Difficulties in managing lymph node tuberculosis. Lung India 2004;21:50

16. Department of Census and Statistics, Sri Lanka. Official Web site of Department of Census and Statistics, Sri Lanka 2015. www. statistics.gov.lk (accessed 15 January 2018).

17. Muluye D, Biadgo B, Woldegerima E et al. Prevalence of tuberculous lymphadenitis in Gondar University Hospital, Northwest Ethiopia. BMC Public Health 2013;13:435. doi:10.1186/1471-2458-13-435.

18. Ali S, Mubeen A, Javed M. Demographic Features of Tuberculous Lymphadenitis Patients. Journal of Sheikh Zayed Medical College 2014;5:730-2.

19. Ozvaran MK, Baran R, Tor M et al. Extrapulmonary tuberculosis in non-human immunodeficiency virus-infected adults in an endemic region. Ann of Thorac Med 2007;2:118121. doi:10.4103/ 1817-1737-33700.

20. Fatmi TI, Jamal Q. A morphological study of chronic granulomatous lymphadenitis with the help of special stains. Pak J Med Sci 2002;18:48-51. 